\title{
Use of Continuous Infusions of Eptifibatide and Cangrelor in Large Vessel Occlusion Acute Ischemic Stroke After Emergent Carotid Artery Stenting
}

\author{
Shaheryar Hafeez ${ }^{\mathrm{a}}$, Pegah Ghamasaee ${ }^{\mathrm{a}, \mathrm{c}}$, Sharmin Amjad ${ }^{\mathrm{b}}$, \\ Colleen Barthol ${ }^{\mathrm{b}}$, Ramesh Grandhi ${ }^{\mathrm{a}}$
}

\begin{abstract}
Placement of carotid stents in the setting of large vessel occlusion (LVO) is sometimes necessary in patients with steno-occlusive disease of the extracranial internal carotid artery (ICA) or ICA dissection. Use of antiplatelet agents is required to prevent in-stent thrombosis; however, after an LVO, a decompressive hemicraniectomy may be necessary. After placement of a carotid stent, a fine balance must be obtained between preventing stent-related thromboembolic complications while also maintaining the possibility of quickly and safely performing a decompressive hemicraniectomy, if indicated. In this case, we discuss the novel use of continuous eptifibatide and cangrelor infusions after carotid stent placement to maintain stent patency, while preserving the option of emergent hemicraniectomy. A 55-yearold man presented with left hemispheric ischemic symptoms due to flow failure from a left ICA dissection. He underwent emergent angiography with angioplasty and stenting of the petrous and ascending cervical segments of the left ICA. The procedure was complicated by an embolization of thrombus to a left middle cerebral artery (MCA) M2 division branch, resulting in an occlusion, which could not be opened. The patient was placed on short acting intravenous antiplatelet agents (eptifibatide infusion for $60 \mathrm{~h}$ and cangrelor infusion for 24 h) for prevention of in-stent thrombosis while under close observation for potential neurologic decline and need for decompressive hemicraniectomy. After $84 \mathrm{~h}$ of observation, the patient did not experience a decline and the antiplatelet infusions were discontinued after he received aspirin and a loading dose of clopidogrel. Intravenous eptifibatide or cangrelor infusions are short-acting antiplatelet options that can be used in patients with acute ischemic stroke from LVO in the setting of ICA stent placement when there exists a potential for decompressive hemicraniectomy.
\end{abstract}

Manuscript submitted April 23, 2020, accepted April 27, 2020

Published online May 30, 2020

aDepartment of Neurosurgery, University of Texas Health Science Center at San Antonio, TX, USA

bDepartment of Pharmacy Services, Neurosurgical Intensive Care Unit, University Health System, San Antonio, TX, USA

${ }^{\mathrm{c} C}$ Corresponding Author: Pegah Ghamasaee, Department of Neurosurgery, University of Texas Health Science Center, 7703 Floyd Curl Drive, MC 7843, San Antonio, TX 78229-3900, USA. Email: ghamasaee@uthscsa.edu

doi: https://doi.org/10.14740/jnr591
Keywords: Internal carotid artery dissection; Middle cerebral artery occlusion; Emergent internal carotid artery stent; Eptifibatide; Cangrelor; P2Y12; GP IIb/IIIa; Ischemic stroke

\section{Introduction}

According to the American Heart Association/American Stroke Association (AHA/ASA), ischemic stroke accounts for $87 \%$ of all strokes in the USA. It is the fifth leading cause of death in the USA and is the leading cause of serious long-term disability [1]. The current acute interventions for acute ischemic stroke include administration of intravenous (IV) recombinant tissue plasminogen activator (rtPA), intra-arterial administration of rtPA, and endovascular mechanical thrombectomy [2].

In up to $10 \%$ of large ischemic strokes involving the middle cerebral artery (MCA), severe post-ischemic cerebral edema may occur, leading to increased intracranial pressure and herniation that carries up to an $80 \%$ mortality risk $[3,4]$. Decompressive hemicraniectomy has been shown in a metaanalysis of seven randomized controlled trials to significantly decrease the mortality of patients suffering from large MCAterritory strokes [5]. Consequently, the AHA/ASA made a class I recommendation stating that decompressive hemicraniectomy in patients $<60$ years of age with unilateral MCA territory infarcts who deteriorate neurologically within $48 \mathrm{~h}$ is effective [6]. Though the optimal trigger or time point for performing surgery is unknown, consensus exists that surgery should be performed before clinical signs of brainstem compression [6].

Carotid artery dissection is a known cause of ischemic stroke and, in the context of endovascular intervention, sometimes requires balloon angioplasty with or without concomitant stent placement. The AHA scientific statement on indications for the performance of endovascular neurointerventional procedures suggests class IIb evidence that endovascular revascularization by intravascular balloon angioplasty and/ or stenting may be considered for patients with symptomatic severe intracranial stenosis ( $70 \%$ luminal narrowing) despite optimal medical therapy [7]. The scientific statement made no comment on the timing of revascularization. In a large multicenter retrospective study, the emergent use of carotid artery stents in combination with anterior circulation thrombectomy 


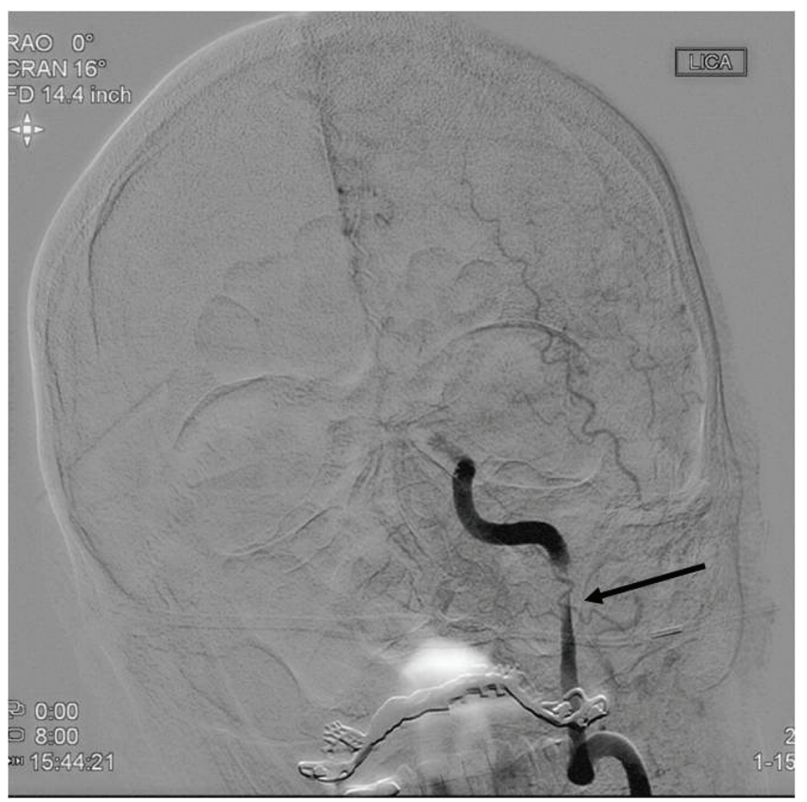

Figure 1. Diagnostic cerebral angiogram (AP view) demonstrating critical left ICA stenosis associated with the dissection in the ascending cervical segment of the left ICA prior to balloon angioplasty and stenting (arrow). ICA: internal carotid artery; AP: anteroposterior.

was effective and safe for the treatment of acute stroke [8]. Placement of a stent requires long-term oral dual antiplatelet therapy (DAPT) with aspirin and a P2Y12 receptor inhibitor to prevent in-stent thrombosis due to the inherent thrombogenicity of intravascular stents. To combat periprocedural thrombosis complications, some clinicians opt to use IV glicoprotein (GP) IIb/IIIa receptor inhibitors as a bridge for transitioning to an oral P2Y12 receptor inhibitor [9].

In the case discussed herein, our patient presented with left hemispheric symptoms from an acute carotid dissection. Balloon angioplasty and stenting were performed within the extracranial left ICA for revascularization, but the procedure was complicated by embolization of thrombus to the inferior division left MCA. IV eptifibatide was administered during the procedure immediately prior to stent placement. However unsuccessful, attempts were made to open up the left M2 inferior division branch. Following the procedure, we did not transition the patient to oral DAPT due to concern that the patient may warrant decompressive hemicraniectomy secondary to infarct and swelling from the left M2 occlusion. We treated the patient with IV eptifibatide and cangrelor until we were confident that the patient did not require surgery. This is the first report on the use of cangrelor, a reversible P2Y12 receptor inhibitor, in the peri-operative period within the neurosurgical literature.

\section{Case Report}

A 55-year-old, right-handed man presented to our institution with left hemispheric symptoms with National Institutes of Health Stroke Scale (NIHSS) score of 22. IV tPA was administered due to the patient presenting within $4.5 \mathrm{~h}$ of symptom

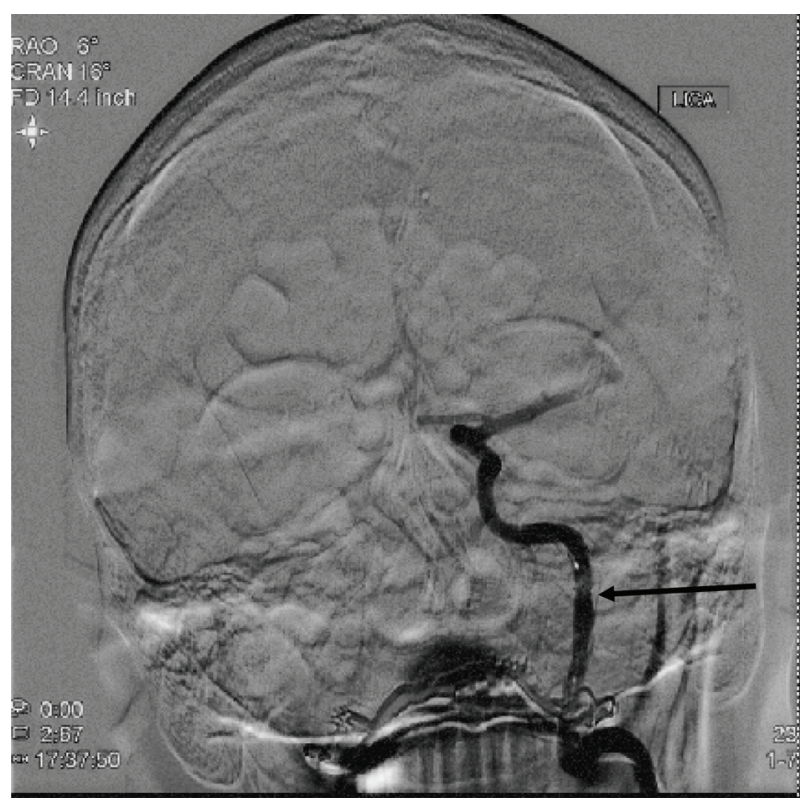

Figure 2. Diagnostic cerebral angiogram (AP view) following balloon angioplasty and stent placement in the ascending cervical segment of the left ICA, showing significantly improved contrast opacification of the artery (arrow). ICA: internal carotid artery; AP: anteroposterior.

onset. Computed tomography (CT) angiography of the head and neck was unrevealing for large vessel occlusion (LVO) within the intracranial circulation but demonstrated a left ICA dissection within the ascending cervical segment of the ICA causing critical stenosis. He was taken for endovascular intervention where balloon angioplasty was performed, and following IV eptifibatide bolus at a dose of $90 \mu \mathrm{g} / \mathrm{kg}$, two $4.5 \times 20$ mm Neuroform EZ ${ }^{\circledR}$ (Stryker Neurovascular, Freemont, CA, USA) stents were placed across the dissected segment of the left ICA (Figs. 1, 2).

After the angioplasty and placement of the stents, thrombus was visualized within the distal aspect of the left MCA M1 division and revascularization of this segment was performed via thrombectomy; however, despite numerous attempts and administration of intra-arterial tPA, the left MCA inferior division M2 artery remained occluded.

The patient was brought to the neurosurgical intensive care unit on an eptifibatide infusion running at $2 \mu \mathrm{g} / \mathrm{kg} / \mathrm{min}$. Follow-up CT imaging demonstrated the expected left MCA infarct involving the temporoparietal lobes resulting from the inferior division M2 occlusion (Fig. 3). Due to the large area of infarct and the patient's young age, he was observed closely for neurologic deterioration or clinical signs of brainstem compression, which would warrant decompressive hemicraniectomy.

The patient was started on a hypertonic saline infusion targeting a serum sodium concentration of $150-155 \mathrm{mmol} / \mathrm{L}$. The patient reached the target within $44 \mathrm{~h}$. We elected not to start the patient on oral DAPT during this period and instead continued the IV eptifibatide infusion for the first 3 days post stroke. At the 3 day mark (the critical time point of maximal cerebral edema with the highest risk for requiring a decom- 


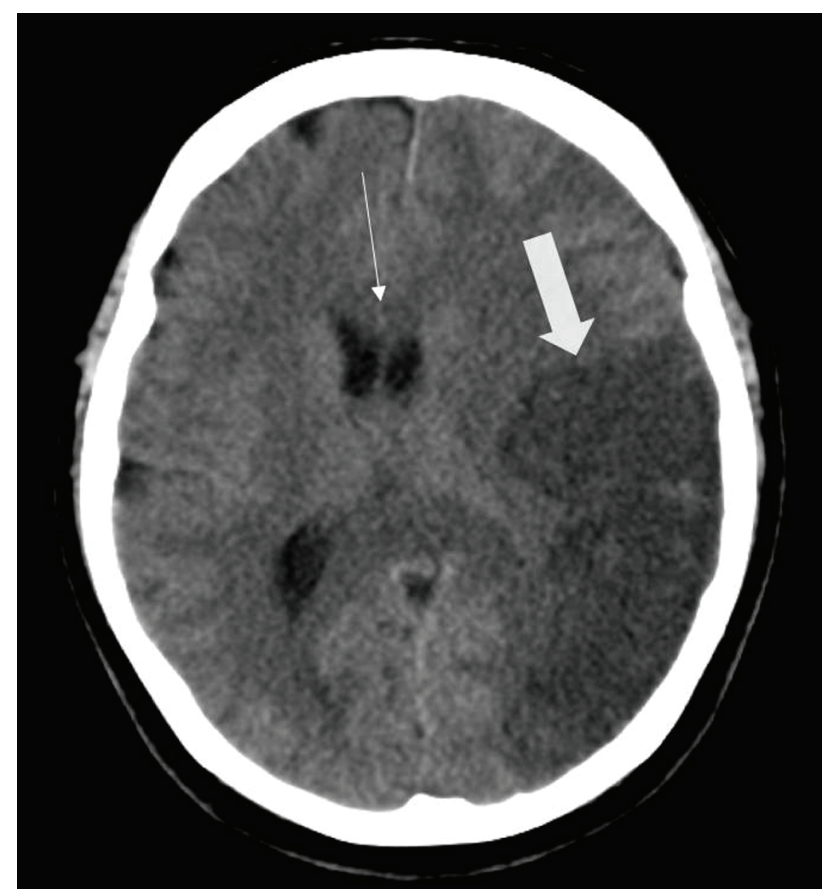

Figure 3. Head CT (axial view) exhibiting hypodensity within the left temporal lobe of the brain, consistent with the left M2 inferior division occlusion (thick arrow). The scan, performed less than $36 \mathrm{~h}$ out from the revascularization procedure, already demonstrates subtle midline shift and mass effect from the area of infarcted brain (thin arrow). CT: computed tomography.

pressive hemicraniectomy), we discontinued the eptifibatide infusion and switched to a continuous infusion of cangrelor at a rate of $4 \mu \mathrm{g} / \mathrm{kg} / \mathrm{min}$ for $24 \mathrm{~h}$. Approximately $84 \mathrm{~h}$ after ictus, after we felt confident that the patient would not require surgical intervention, the patient was loaded with $300 \mathrm{mg}$ clopidogrel and $325 \mathrm{mg}$ aspirin and the cangrelor infusion was subsequently discontinued. CT angiography performed 6 days after presentation revealed patency of the carotid stents with asymptomatic petechial hemorrhage of the infarction. Over the next several days, the patient's clinical exam slowly improved and he was discharged to inpatient rehabilitation.

\section{Discussion}

The occurrence of thrombus formation immediately after carotid arterial injury and stenting and the need for emergent decompressive hemicraniectomy provide the rationale for early IV antiplatelet therapy. The accepted practice of DAPT was not followed immediately post-stent placement in this case because of the risk of requirement of a decompressive hemicraniectomy. Although there is no literature on the matter, there is a general consensus among neurosurgeons to not perform cranial surgery on patients taking DAPT due to the possibility of uncontrolled bleeding during surgery. Our patient was managed with two short acting IV antiplatelet agents, eptifibatide and cangrelor. Eptifibatide is a cyclic peptide derivative that reversibly binds the GP IIb/IIIa receptors, with subsequent in- hibition of platelet aggregation and thrombus formation. Eptifibatide is Food and Drug Administration (FDA) approved for treatment of acute coronary syndrome managed medically or with percutaneous coronary intervention (PCI), and management of PCI with or without intracoronary stenting to reduce the risk of acute cardiac ischemic events (death and/ or myocardial infarction (MI)). Additionally, cangrelor, a nonthienopyridine adenosine triphosphate analogue, is the only IV antagonist of the P2Y12 receptor exhibiting rapid, potent, predictable, and reversible platelet inhibition with quick offset of effect [10]. Cangrelor is currently indicated as an adjunct to PCI to reduce the risk of MI, repeat coronary revascularization, and stent thrombosis in patients who have not been treated with a P2Y12 platelet inhibitor and are not being given a GP IIb/IIIa inhibitor [11].

The platelet GP IIb/IIIa receptor is the final common pathway leading to the binding of fibrinogen to the activated platelet GP IIb/IIIa and thrombus formation. GP IIb/IIIa is therefore a logical therapeutic target for prevention of ischemic complications during percutaneous coronary procedures. Despite the benefits seen in trials with coronary intervention using these agents, limited data has adapted the use of GP IIb/IIIa inhibitors in carotid arterial stenting. Qureshi et al reported on the safety of eptifibatide as an adjunct to carotid angioplasty and stent placement (CAS) for extracranial carotid artery stenosis [12]. Eptifibatide was administered IV as two $180 \mu \mathrm{g} / \mathrm{kg}$ bolus doses, and then as a $2 \mu \mathrm{g} / \mathrm{kg} / \mathrm{min}$ infusion for 20 - $24 \mathrm{~h}$. Among the 25 patients who underwent the procedure, no intracerebral hemorrhage and one minor ischemic stroke occurred during the 1-month follow-up period. One episode of major bleeding from the femoral insertion site required surgical repair and blood transfusion. The authors concluded that eptifibatide administered as an adjunct to CAS seems to be safe. Eptifibatide's pharmacokinetic parameters include immediate onset of action after initial bolus ( $>80 \%$ inhibition of adenosine diphosphate (ADP)-induced aggregation achieved 5 min after bolus) with maximal effect within $1 \mathrm{~h}$. Platelet function is restored $4-8 \mathrm{~h}$ following discontinuation, and its elimination half-life is $2.5 \mathrm{~h}$. Finally, it is recommended to discontinue eptifibatide at least 2 - $4 \mathrm{~h}$ prior to coronary artery bypass graft surgery [13].

Most reports of GP IIb/IIIa inhibition during CAS feature the use of abciximab, a monoclonal antibody with a half-life of $10 \mathrm{~min}$, but an effect on platelets lasting for almost $48 \mathrm{~h}$. Kapadia et al performed a study in 151 consecutive, highsurgical-risk patients: 128 received abciximab and 23 controls were given only heparin. There were more adverse procedural events in the control group ( $8 \%$; one major stroke and one neurological death) compared with the abciximab group (1.6\%; one minor stroke and one retinal infarction; $\mathrm{P}=0.05)$. At 30 day follow-up, one patient presented with delayed intracranial hemorrhage (ICH) in the abciximab group [14]. Qureshi et al prospectively examined 70 patients: 37 given abciximab as an adjunct to high-risk carotid stenting versus 33 patients with standard intraprocedural heparinization. The 3\% frequency of ischemic stroke in the high-risk patients given IV abciximab was lower than controls; however, the benefit was lost because of the higher rate $(5 \%)$ of ICH [15]. Further studies are needed to determine the appropriate role of abciximab in this patient population. 
Similarly, there is a paucity of data on the use of continuous infusion cangrelor in the setting of CAS in acute ischemic stroke. Following a cangrelor IV bolus and infusion, platelet inhibition occurs within minutes and the average half-life is 3 - $6 \mathrm{~min}$. In healthy volunteers, a $30 \mu \mathrm{g} / \mathrm{kg}$ bolus followed by a $4 \mu \mathrm{g} / \mathrm{kg} / \mathrm{min}$ infusion provided extensive inhibition of platelet aggregation 2 min after the bolus dose, and $80 \%$ of subjects returned to near-baseline platelet activity within $60 \mathrm{~min}$ [16]. These pharmacokinetic properties of cangrelor make it an attractive option to consider for bridging patients to surgery in whom discontinuation of antiplatelet therapy, particularly a P2Y12 inhibitor, can lead to catastrophic consequences while preserving normal hemostasis at the time of surgery. Pharmacodynamic studies demonstrate a substantial interaction when a thienopyridine loading dose is administered during cangrelor infusion. Specifically, the antiplatelet effect of a clopidogrel loading dose is substantially attenuated when given in the midst of the infusion (and therefore will not provide the anticipated P2Y12 inhibition once the infusion is discontinued), likely because the labile, short lived active metabolite of clopidogrel cannot bind to the P2Y12 receptor when it is occupied by cangrelor [17]. Therefore, a clopidogrel loading dose should be administered at the end of the cangrelor infusion.

Given the much shorter half-life of cangrelor and potential need for emergent surgery, we transitioned our patient from the eptifibatide infusion to cangrelor, followed by a clopidogrel loading dose and maintenance therapy with clopidogrel and aspirin daily. Our case is novel because we demonstrated that long-term use of continuous IV infusions of eptifibatide $(60 \mathrm{~h})$ and cangrelor ( $24 \mathrm{~h}$ ) can be done safely and effectively while preserving the option of emergent cranial surgery.

\section{Acknowledgments}

None to declare.

\section{Financial Disclosure}

None to declare.

\section{Conflict of Interest}

None to declare.

\section{Informed Consent}

Informed consent was obtained.

\section{Author Contributions}

PG and SA prepared the manuscript. SH, RG and $\mathrm{CB}$ performed revisions and approved the final version for submission.

\section{Data Availability}

The data supporting the findings of this study are available from the corresponding author upon reasonable request.

\section{References}

1. Benjamin EJ, Blaha MJ, Chiuve SE, Cushman M, Das SR, Deo R, de Ferranti SD, et al. Heart disease and stroke statistics-2017 update: a report from the American Heart Association. Circulation. 2017;135(10):e146-e603.

2. Chaudhary N, Pandey AS, Gemmete JJ. Intervention in acute cerebral ischaemic stroke: a review of the role of pharmacological therapies and intra-arterial mechanical thrombectomy devices. Postgrad Med J. 2011;87(1032):714-723.

3. Hacke W, Schwab S, Horn M, Spranger M, De Georgia M, von Kummer R. 'Malignant' middle cerebral artery territory infarction: clinical course and prognostic signs. Arch Neurol. 1996;53(4):309-315.

4. Berrouschot J, Sterker M, Bettin S, Koster J, Schneider D. Mortality of space-occupying ('malignant') middle cerebral artery infarction under conservative intensive care. Intensive Care Med. 1998;24(6):620-623.

5. Alexander P, Heels-Ansdell D, Siemieniuk R, Bhatnagar N, Chang Y, Fei Y, Zhang Y, et al. Hemicraniectomy versus medical treatment with large MCA infarct: a review and meta-analysis. BMJ Open. 2016;6(11):e014390.

6. Wijdicks EF, Sheth KN, Carter BS, Greer DM, Kasner SE, Kimberly WT, Schwab S, et al. Recommendations for the management of cerebral and cerebellar infarction with swelling: a statement for healthcare professionals from the American Heart Association/American Stroke Association. Stroke. 2014;45(4):1222-1238.

7. Meyers PM, Schumacher HC, Higashida RT, Barnwell SL, Creager MA, Gupta R, McDougall CG, et al. Indications for the performance of intracranial endovascular neurointerventional procedures: a scientific statement from the American Heart Association Council on Cardiovascular Radiology and Intervention, Stroke Council, Council on Cardiovascular Surgery and Anesthesia, Interdisciplinary Council on Peripheral Vascular Disease, and Interdisciplinary Council on Quality of Care and Outcomes Research. Circulation. 2009;119(16):2235-2249.

8. Behme D, Mpotsaris A, Zeyen P, Psychogios MN, Kowoll A, Maurer CJ, Joachimski F, et al. Emergency stenting of the extracranial internal carotid artery in combination with anterior circulation thrombectomy in acute ischemic stroke: a retrospective multicenter study. AJNR Am J Neuroradiol. 2015;36(12):2340-2345.

9. Qureshi AI. Adjunctive use of platelet glycoprotein IIb/ IIIa inhibitors for carotid angioplasty and stent placement: time to say good bye? J Endovasc Ther. 2003;10(1):4244.

10. Ferreiro JL, Ueno M, Angiolillo DJ. Cangrelor: a review on its mechanism of action and clinical development. Expert Rev Cardiovasc Ther. 2009;7(10):1195-1201. 
11. KENGREAL [package insert]. Cary, NC: Chiesi USA, Inc; 2015.

12. Qureshi AI, Siddiqui AM, Hanel RA, Xavier AR, Kim SH, Kirmani JF, Boulos AS, et al. Safety of high-dose intravenous eptifibatide as an adjunct to internal carotid artery angioplasty and stent placement: a prospective registry. Neurosurgery. 2004;54(2):307-316; discussion 316-307.

13. Eptifibatide [package insert]. Whitehouse Station, NJ: Merck \& Co. Inc.; 2014.

14. Kapadia SR, Bajzer CT, Ziada KM, Bhatt DL, Wazni OM, Silver MJ, Beven EG, et al. Initial experience of platelet glycoprotein IIb/IIIa inhibition with abciximab during carotid stenting: a safe and effective adjunctive therapy. Stroke. 2001;32(10):2328-2332.
15. Qureshi AI, Suri MF, Ali Z, Kim SH, Lanzino G, Fessler $\mathrm{RD}$, Ringer AJ, et al. Carotid angioplasty and stent placement: a prospective analysis of perioperative complications and impact of intravenously administered abciximab. Neurosurgery. 2002;50(3):466-473; discussion 473-465.

16. Akers WS, Oh JJ, Oestreich JH, Ferraris S, Wethington M, Steinhubl SR. Pharmacokinetics and pharmacodynamics of a bolus and infusion of cangrelor: a direct, parenteral P2Y12 receptor antagonist. J Clin Pharmacol. 2010;50(1):27-35.

17. Price MJ, Teirstein PS. Dynamics of platelet functional recovery following a clopidogrel loading dose in healthy volunteers. Am J Cardiol. 2008;102(6):790-795. 